livraisons

d'Histoire

de l'Architecture

\section{Livraisons de l'histoire de l'architecture}

16 | 2008

Grands chantiers et matériaux

\title{
Architecture : les matériaux d'une histoire
}

Architecture: materials for its history

Architektur : die Materialien zu einer Geschichte

\section{Nicolas Reveyron}

\section{(2) OpenEdition}

\section{Journals}

Édition électronique

URL : http://journals.openedition.org/lha/192

DOI : 10.4000/lha.192

ISSN : 1960-5994

Éditeur

Association Livraisons d'histoire de l'architecture - LHA

Édition imprimée

Date de publication : 10 décembre 2008

Pagination : 139-150

ISSN : 1627-4970

Référence électronique

Nicolas Reveyron, «Architecture : les matériaux d'une histoire », Livraisons de l'histoire de l'architecture [En ligne], 16 | 2008, mis en ligne le 23 avril 2014, consulté le 19 avril 2019. URL : http:// journals.openedition.org//ha/192 ; DOI : 10.4000/Iha.192

Ce document a été généré automatiquement le 19 avril 2019

Tous droits réservés à l'Association LHA 


\title{
Architecture : les matériaux d'une histoire
}

\author{
Architecture: materials for its history \\ Architektur : die Materialien zu einer Geschichte
}

Nicolas Reveyron

10 décembre 2010

Cette journée d'étude a parfaitement rempli sa mission. En «traitant de l'économie et du mode d'organisation du chantier, du Moyen Âge à la fin du XIX siècle en Europe », les intervenants ont tous confirmé l'importance prise dans l'histoire de l'architecture par l'étude des matériaux de construction. Car, depuis les calcaires des églises troyennes tardo-gothiques présentés par Cédric Roms, jusqu'aux bétons armés décrits par Georges Beisson, en passant par tous les marbres rutilants de l'âge classique, ces derniers sont au cœur des problématiques du chantier. Le fait se vérifie pour l'ensemble du Moyen Âge, période où je m'autoriserai à puiser des exemples complémentaires.

\section{Le matériau, source de connaissances}

3 L'intérêt pour les matériaux lithiques est, en France, une tendance lourde, il faut le souligner. Tendance lourde de la culture populaire, comme en témoigne le vocabulaire courant touchant le patrimoine national. Ramassant d'instinct en une formule lapidaire la complexité d'une problématique d'histoire de l'art, la vox populi désigne ainsi volontiers par « vieilles pierres » des monuments que la datation de leurs appareils nous rend quasi contemporains comparativement à l'âge géologique de leur matière. Tendance lourde aussi de la recherche scientifique, du moins en ce qui concerne le Moyen Âge. Avec Montesquieu, en effet, le siècle des Lumières avait déjà établi une étroite corrélation entre la géographie physique, notamment les ressources du sous-sol, et le développement des civilisations. Dans cette lignée, au début du XIX siècle, les premiers archéologues médiévistes, formés aux sciences de la nature ${ }^{1}$, ont trouvé dans l'identification des pierres 
et dans l'analyse des mises en œuvre une voie royale pour comprendre les édifices du Moyen Âge et pour en restituer l'histoire².

Aujourd'hui, l'archéologie du bâti a débordé le champ traditionnel des pierres et des appareils, pour s'intéresser à tous les matériaux qui entrent dans la construction. Les bois ${ }^{3}$, la chaux, les sables, les mortiers ${ }^{4}$, les pièces de fer ${ }^{5}$, le plomb, les verres ${ }^{6}$, les terres cuites architecturales ${ }^{7}$, etc. sont de véritables « archives monumentales » que les sciences dites dures (géologie, sciences de la nature, physique, chimie ...) et les analyses techniques, notamment celle des traces de production et de montage, permettent de décrypter ${ }^{8}$. Au-delà des modes de production et des savoir-faire, cette science encore jeune en a tiré des données essentielles sur l'organisation des chantiers médiévaux, depuis l'outillage ou l'usage de machines, jusqu'à la hiérarchisation des travailleurs et à la répartition des tâches, voire l'élaboration des techniques ou la transmission des savoirs9. L'apport est considérable. Il restitue une réalité médiévale dont les sources écrites et iconographiques, très lacunaires et idéologiquement orientées, avaient transmis une image déformée. Le chantier du Moyen Âge a retrouvé sa « modernité ». Ainsi, la théorie $\mathrm{du}$ «mensonge architectural» élaborée par John Ruskin ${ }^{10}$ se découvre une lointaine origine dans la dénonciation par Jean Mignot, à l'extrême fin du XIV ${ }^{e}$ siècle, de l'utilisation du fer dans le chantier de la cathédrale de Milan ${ }^{11}$. De même, la rationalisation des modes de production, étudiée sur le chantier de la cathédrale de Reims par Dieter Kimpel ${ }^{12}$, annonce précocement celle que l'architecte moderne imposera progressivement.

5 La rationalisation de l'organisation du chantier et du travail des constructeurs, justement, Jean-Michel Leniaud nous a rappelé qu'elle devait être au centre des recherches sur l'architecture moderne et contemporaine. La tout récente publication de L'Enquête $d u$ Régent sur les ressources naturelles du royaume vient à point nommé confirmer que ce mouvement n'a pas attendu le Révolution ${ }^{13}$. Et les difficultés d'approvisionnement en marbre des chantiers de Louis XIV, décrites par Pascal Julien, n'ont sans doute pas peu contribué à la prise de conscience d'une telle nécessité. La pléthore de documents écrits, graphiques ou sonores (projets, plans, élévations, prix-faits, traités techniques, correspondances, jugements esthétiques, expertises, rapports d'étude, articles de journaux, interview ...) place les matériaux sous les faisceaux convergents de puissants éclairages. Mais cette débauche d'écrits n'exclut pas pour autant l'approche archéologique. L'analyse que Guillaume Fonkenell a proposé des matériaux mis en œuvre dans l'arc du Carrousel, notamment des colonnes vraisemblablement remployées, confirme la nécessité des études de terrain, même pour des périodes très tardives. Très beau thème de recherche, d'ailleurs, qui traverse l'histoire de l'architecture du Moyen Âge à notre époque, avec une permanence des problématiques qui force l'intérêt (cf. infra ).

6 Cette rationalisation ne constitue pas un mouvement uniforme. Il faut l'envisager à deux niveaux. D'une part, celui de chaque chantier, lieu particulier où se sont toujours élaborées empiriquement les normes pratiques de chaque construction. D'autre part, à partir du XIXe siècle et en France, celui de l'État, promoteur de normes nationales à visée universelle, comme le système métrique, et garanties par le savoir des scientifiques et des ingénieurs (autrement dit rationalisées). Les éventuelles discordances entre les deux niveaux ont eu un impact le plus souvent négligeable, voire inexistant, comme le montre le chantier du phare d'Armen décrit par Jean-Christophe Fichou, entreprise locale d'intérêt national. Il en est allé tout autrement dans certains domaines, comme la 
restauration des monuments historiques. Celle-ci, en effet, associe deux réalités discordantes, d'une part la norme nationale, qui tend à uniformiser à l'échelle du pays les références techniques les plus récentes, d'autre part l'édifice ancien, pétri de particularismes, parce que construit avec des matériaux locaux et suivant des techniques spécifiques. La confrontation de ces deux niveaux culturels n'est pas allée sans difficultés. Prenons la question du choix des pierres destinées à remplacer les blocs défectueux, problème qu'Arnaud Timbert a évoqué à travers les travaux d'Eugène Viollet-Le-Duc à Vézelay. L'inaccessibilité des carrières médiévales a privé les architectes des matériaux utilisés par leurs devanciers. Elle les a contraints à employer des qualités de pierre approchantes, choisies d'après les nomenclatures nationales. Or, il n'existe pas d'équivalence absolue d'une qualité à l'autre. Telle ou telle caractéristique mécanique ou chimique différencie drastiquement deux pierres apparemment proches. Par ailleurs, le marché des matériaux lithiques ne pouvait alors s'encombrer de ce qui pouvait apparaître comme des nuances et que l'état des connaissances scientifiques ne permettait pas même d'apprécier à sa juste mesure. Pour toutes ces raisons, les pierres de substitution ont souvent révélé, avec le temps, des incompatibilités désastreuses avec les matériaux d'origine, occasionnant des dégâts lourds.

\section{Le matériau dans l'économie du chantier}

$7 \quad$ Les communications de cette journée ont insisté sur trois aspects de l'interaction entre le matériau et le chantier: l'aspect économique, l'aspect technique et l'aspect esthétique. Question systématiquement abordée, le coût de la pierre est venu rappeler que le chantier relève aussi de l'économique. Les approvisionnements en matériaux de proximité, voire en matériaux de ramassage, tous d'un faible coût de production et de transport, caractérisent la construction courante, mais aussi des chantiers majeurs faiblement dotés, comme l'a montré par exemple Isabelle Parron pour les cathédrales savoyardes du $\mathrm{XI}^{e}$ siècle ${ }^{14}$. Le remploi de vieux matériaux est, du Moyen Âge jusqu'au XXI ${ }^{e}$ siècle, une autre réponse aux difficultés financières. Que ce soit à Milan au $\mathrm{IV}^{\mathrm{e}}$ siècle, où les briques de récupération, entières ou brisées, sont à la base de l'opus spicatum ambrosien ${ }^{15}$, à l'abbatiale de La Chaise-Dieu, où les mortiers du vieil édifice ont été remployés après broyage dans les mortiers de l'église en construction au XIV siècle $^{16}$, ou dans les chantiers de la seconde moitié $\mathrm{du} \mathrm{XX}^{\mathrm{e}}$ siècle, où le béton des démolitions est réduit en granulat réutilisable ${ }^{17}$, le remploi permet chaque fois de réduire deux postes budgétaires importants: l'achat de matériau et le transport des déchets. Mais quand il s'agit de pierres de très haute qualité, le remploi a des fonctions autres qu'économiques. L'usage de matériaux antiques ou de beaux matériaux - le Moyen Âge en a fait de ces deux expressions des synonymes -, qui s'avèrent souvent onéreux puisqu'il s'agit le plus souvent de marbres, était surtout destiné à fonder une esthétique antiquisante et à affirmer une filiation avec les origines antiques de l'institution maîtresse d'œuvre ${ }^{18}$. La réponse couramment apportée par le Moyen Âge aux difficultés d'approvisionnement en marbres a été les matériaux de substitution, principalement les pierres imitant des marbres, comme les « équivalents marbres » de Suger ${ }^{19}$, les choins de la Renaissance du XII ${ }^{\mathrm{e}}$ siècle à Lyon ou Vienne ${ }^{20}$, les pierres marbrières des Pyrénées ou des Alpes, les calcaires fins et colorés polissables («marbre noir» de Purbeck et de Corfe (Angleterre), de Tournai ou de Liège, pierre bleu de Wallonie ...) ou les magnifiques pièces d'architecture moirées des pays germaniques, tirées des concrétions de calcite accumulées dans les 
aqueducs antiques ${ }^{21}$. En revanche, les enduits peints imitant le marbre, abondamment employés dans l'architecture romane de moellon, dissocient l'apparence et la matière. Mais ils témoignent d'un extraordinaire savoir-faire : pour obtenir dans la matière même l'aspect du marbre poli, le maçon doit, par de nombreuses passes, serrer le mortier pour en extraire la plus grande quantité d'eau, jusqu'à ce qu'il acquiert cet aspect faïencé caractéristique ; la peinture n'intervient que pour figurer les veines des marbres colorés.

Les matériaux onéreux sont donc associés à des chantiers prestigieux. L'emploi de marbres, par exemple, vise à exalter la puissance du commanditaire, comme l'ont montré à l'envi Pascal Julien et Sophie Mouquin. Puissance esthétique, si l'on peut dire, qui s'exprime dans la variété des couleurs et la subtilité des compositions. Il s'agit là d'un thème déjà largement développé aux époques carolingiennes et romanes. Les Miracles de saint Magloire, par exemple, texte écrit en Bretagne au IX ${ }^{\mathrm{e}}$, décrivent les colonnes de marbre d'un temple antique, «les unes blanches veinées de pourpre, les autres pourpre tachetées de blanc, pour que la diversité des couleurs charme de son spectacle varié le cœur des spectateurs $»^{22}$. Puissance financière surtout, affichée en pleine architecture par la masse de matériaux de prix dont le coût d'achat est renchéri au-delà du raisonnable par celui du transport. De fait, toute l'histoire de l'architecture occidentale est émaillée de ces lointains voyages d'approvisionnement, depuis le transport jusqu'à Rome de colonnes de marbre, luxe violemment dénoncé par Pline l'Ancien, ou d'obélisques égyptiens, opération pour laquelle Caligula fit construire une trirème hors gabarit, restée dans les annales et au fond du port d'Ostie. Le Moyen Âge a largement exploité ce filon de gloire : Charlemagne faisant quérir des marbres à Rome pour Aix-la-chapelle, geste répété 200 ans plus tard par l'abbé Didier pour l'abbaye du Mont Cassin, saint Odilon faisant ramener des marbres de Provence " par le cours furieux de la Durance » (première moitié $\mathrm{du} \mathrm{XI}^{\mathrm{e}}$ ), Suger projetant de rapporter des marbres de Rome par la Méditerranée, l'Atlantique et la Seine jusqu'à Saint-Denis... Mais il faut rester prudent. Certes, l'usage de pierres de qualité donne la mesure du niveau financier du chantier. Quand, au début du $\mathrm{XI}^{\mathrm{e}}$ siècle, l'abbé Gauzlin faisait venir des blocs de calcaire fin du Nivernais pour son abbaye de Fleury (Saint-Benoît-sur-Loire), il investissait dans un matériau plus onéreux et plus beau que le calcaire local, pourtant abondant. Mais l'importation de «belles pierres » a créé des courants commerciaux susceptibles de favoriser l'abaissement des coûts. La pierre de Gobertange, dans la Belgique actuelle, a fait l'objet d'un commerce depuis l'époque romaine ${ }^{23}$ et, dès le $\mathrm{XI}^{e}$ siècle, la pierre de Caen a été exportée d'abord jusqu'en Bretagne, puis outre Manche dès la conquête normande ${ }^{24}$.

Le transport de pierre appartient à l'histoire de l'art pour d'autres raisons. Contre l'idée d'une architecture médiévale strictement vernaculaire, fille de la terre et des hommes de la région, les chercheurs de la seconde moitié du XIXe siècle ont découvert dans les importations de pierre ou de produits finis une autre source d'influence majeure. Camille Enlart écrivait au début du XX $\mathrm{X}^{\mathrm{e}}$ siècle :

Nous avons vu que la qualité des matériaux dont dispose une contrée exerce généralement une influence sur le style de ce pays[...], d'autre part, les importations de matériaux ont coïncidé souvent avec des importations de style; au $\mathrm{VI}^{\mathrm{e}}$ siècle, des chapiteaux sont sortis tout travaillés des carrières de marbre de l'Empire byzantin ; aux XI ${ }^{\mathrm{e}}$ et $\mathrm{XII}^{\mathrm{e}}$, des colonnes, des tombes, des fonts baptismaux de Tournai, ont répandu les formes lombardes ou rhénanes en usage dans cette ville, tandis que du port de Boulogne s'exportaient jusqu'à Bruges de belles pierres blanches de Marquise (calcaire oolithique) sculptées dans des ateliers qui conservaient, au contraire, les traditions gallo-romaines ${ }^{25}$. 
10 Le voyage intègre alors la problématique du modèle et de la transmission des formes, assurée par l'objet déplacé autant que par le dessin, les visites de chantier ou la formation en atelier. C'est une constante de l'histoire de l'art, qui se vérifie aux époques de grands bouleversements. Par exemple, l'exportation par des ateliers italiens de la Renaissance de chapiteaux, de pilastres et de niches sur toute l'Europe a été un des vecteurs déterminants de la propagation du style, comme cela a été le cas pour les magnifiques aménagements Renaissance du château de La Calahorra (Andalousie), commandés au début du XVI ${ }^{\mathrm{e}}$ siècle par le marquis Rodrigo Diaz de Vivar y Mendoza. Mais au XII ${ }^{\mathrm{e}}$ siècle, déjà, les cloîtres cosmatesques étaient produits en atelier, à Rome, et livrés en pièces détachées, dans la ville et jusqu'à Subiaco ${ }^{26}$.

11 Cette journée a peu abordé les problématiques techniques, sinon pour l'ingénierie du phare d'Armen, la production du béton ou l'extraction des marbres pyrénéens. L'intérêt pour les carrières est un aspect récent de l'histoire de l'architecture. Le Moyen Âge s'était montré déjà sensible à la question. En 1911, Victor Mortet citait, dans son Recueil de textes relatifs à l'histoire de l'architecture, un extrait du Tractatus de ecclesie S. Petri (XI $\left.{ }^{\mathrm{e}}\right)$ où l'auteur identifie l'origine des pierres retrouvées en fouilles pour la construction de la nouvelle église $^{27}$. À un autre niveau culturel, l'auteur du Charroi de Nîmes, chanson de geste des environs de 1140, donne, à propos des sites de Vésène et Lavardin traversés par le comte Guillaume, cette précision significative: "d'où fut extraite la pierre / qui servit à construire les tours de Nîmes ${ }^{28}$ ". Moins spectaculaires que le transport de pierres ou de colonnes, les carrières sont entrées dans l'histoire de l'architecture par la porte de la localisation des sources d'approvisionnement. Les recherches ont été menées à partir des années 1980 soit par des historiens de l'économie ${ }^{29}$, soit par des géologues ${ }^{30}$, soit par des archéologues spécialisés. Elles ont abordé des problématiques portant sur la propriété des carrières, l'économie des sites de production ou le rôle des carriers dans l'organisation de la construction (livraison de blocs bruts, épannelés ou presque achevées, ces derniers réduisant d'autant le coût du transport ${ }^{31}$ ).

12 La question de la taille de pierre est, dans l'optique qui nous intéresse, l'indice technique sans doute le plus remarquable, parce que la visibilité, la banalisation ou la dissimulation des traces d'outils évoluent avec la conception que chaque époque stylistique se fait du travail de terrain ${ }^{32}$. Les archéologues médiévistes s'y sont intéressés très tôt. " La nature de la taille, écrit Eugène Viollet-Le-Duc à l'article « Taille » de son Dictionnaire raisonné (t. VIII, p. 1-6), est un des moyens les plus certains de reconnaître la date d'une construction; mais dès le XII ${ }^{\mathrm{e}}$ siècle, les diverses écoles de tailleurs de pierre ont des procédés qui leur appartiennent, et qu'il est nécessaire de connaître pour éviter les confusions. Ainsi, certaines provinces n'ont jamais adopté la laye ou la bretture, ou n'ont employé cet outil que très tard. Des tailleurs de pierre ne se sont servis que du ciseau étroit ou large ; quelques contrées ont employé de tout temps le marteau sans dent, avec plus ou moins d'adresse. Mais le Moyen Âge, plus précisément l'architecture romane, se distingue des autres époques par un usage encore mal expliqué : la «taille décorative ». Il s'agit de formes ornementales délibérément données aux traces d'outils, bien attestées dans l'art roman provençal ${ }^{33}$. Cette attitude esthétique place le tailleur de pierre dans une position très originale sur le chantier, comme s'il jouissait d'une liberté que personne ne connaîtra après le XIII ${ }^{\mathrm{e}}$ siècle. Les marques lapidaires, en usage en Occident depuis l'Antiquité grecque et romaine, posent la même question sur la publicité des travailleurs du chantier. On sait qu'elles avaient pour fonction d'identifier soit le travail d'un tailleur de pierre, soit la position du bloc dans le bâti, soit la livraison d'un $\operatorname{lot}^{34}$. Les formes 
utilisées, notamment les noms en toutes lettres de l'architecture romane provençale, la qualité des gravures et les dimensions parfois considérables des marques médiévales suggèrent les mêmes hypothèses que les tailles décoratives. La persistance à l'époque moderne de belles marques, nettement visibles dans le bâti, peut surprendre. Il est vrai qu'au XVIII ${ }^{e}$ siècle, à l'inverse, on a fréquemment utilisé des marques gravées non pas sur la surface du bloc, mais sur une saillie laissée brute à cet effet, et donc facile à faire disparaître après la pose, par bûchage. Mais, parallèlement, le $\mathrm{XX}^{\mathrm{e}}$ siècle a redécouvert le pouvoir ornemental des marques lapidaires: l'architecte qui, dans les années 1935, a construit l'église San Giorgio al Corso, à Reggio de Calabre, a parsemé les parements extérieurs de marques de grandes dimensions et d'une belle gravure, toutes inventées, comme une citation de l'art médiéval ${ }^{35}$.

\section{Les matériaux entre narration et illusion}

13 Cette journée s'inscrira sans doute comme un moment emblématique dans l'évolution actuelle de l'histoire de l'architecture. Se sont retrouvées enfin au centre des préoccupations, des questions que la hiérarchie des genres et des problématiques, le manque d'intérêt des scientifiques, ou une décence mal placée, abandonnaient habituellement aux marges de la recherche, ou aux poubelles de l'histoire. Ce repositionnement épistémologique est, on le sait, un des effets les plus novateurs de l'influence du transcendantalisme américain sur la recherche occidentale.

Il est aujourd'hui devenu possible, non pas même d'aborder seulement ou d'évoquer, mais bien de traiter et d'analyser des relations jugées alors aussi improbables que «la rencontre fortuite sur une table de dissection d'une machine à coudre et d'un parapluie » : rapports entre l'architecture et la liturgie, évoqués à la chapelle du château de Versailles, question longtemps bannie de la recherche publique par notre République laïque ${ }^{36}$, entre l'architecture et les dépassements de budget (c'est Vauban stigmatisant les gabegies du chantier de Versailles!), entre l'architecture et les détournements de matériaux, phénomène aussi constant que le remploi dans l'histoire de l'art ${ }^{37}$, entre l'architecture et la rationalisation des travaux, entre l'architecture et les jugements de cour, qui changent de ton aussi vite que la mode, entre l'architecture et la communication, qui a fait entendre son bruissement et sonner ses trompettes dans les aménagements de la fontaine de Grenelle, présentés par Domnique Massounie... Mais la communication n'est-elle pas déjà une partie de l'histoire ? Car tout ce qui nous a été dit aujourd'hui, tous ces portraits peints sur le vif, ces événements mondains et ces tractations de coulisse, les hauts faits ou les petits arrangements des entrepreneurs, etc. ont visé d'abord à réécrire la geste du chantier, le récit de la construction. Comme l'a écrit Paul Veyne, « une page d'histoire ressemblera toujours plus à une page de narration qu'à une page de physique $»^{38}$. Nous plaçons nos pas dans ceux des chroniqueurs du Moyen Âge et des auteurs de romans comme la Chanson de Girard de Roussillon, pour le chantier de Vézelay, ou des Quatre fils Aymon, pour celui de Cologne. Cette attitude est essentielle: une partie de la beauté de l'édifice appartient au chantier et tient dans le génie technique ${ }^{39}$. Mais cette beauté-là n'en est transmise que par les mots et par les impressions produites. Fixons en la réalité, avant que la légende ne s'en empare, comme elle s'est emparée de la construction des pyramides ou du phare d'Alexandrie.

Dans cette fresque haute en couleurs s'est glissée, subrepticement, sans invitation, une question nécessaire qui confine à une phénoménologie de l'architecture. Malgré les 
apparences, les matériaux entretiennent avec l'édifice une relation pour le moins complexe, parce qu'ils relèvent à la fois de l'architecture et de l'architectonique. Quoiqu'ils en soient la substance, par exemple, ils affichent à la fois un âge propre, indépendamment de la chronologie des travaux, et un âge " architecturé ». Ce n'est pas la naissance de l'arbre, mais la date de l'abattage et de l'emploi qui importe. Ce n'est pas la formation des roches, mais la taille du bloc qu'il faut dater. Ce n'est pas la matière, mais bien l'élément façonné qui entre dans la chronologie de la construction. En revanche, c'est bien la matière, et non plus le matériau, qui importe à l'esthétique, par ses qualités intrinsèques, mais aussi dans leur mise en forme par le spécialiste (en polissant les marbres, il en exalte leur beauté culturelle), et dans l'élaboration du projet par l'architecte (qui joue sur les couleurs, les contrastes, les dispositions rythmées ...). Dans ce concert d'intentions et de réalisations, la restauration des monuments opère un renversement épistémologique des valeurs communes. Quand l'architecte remplace des blocs dans un édifice, il en change la substance pour en préserver le dessin. Ce n'est pas tant la pierre qui perdure que, plutôt, l'arrête de la baie ou le galbe de la colonne : le bloc est remplacé, parce que sa forme s'est émoussée et bien qu'il remplisse encore sa fonction dans la stabilité du bâtiment. Avec les reconstructions d'après guerre, la substance des bâtiments a été plus gravement atteinte, comme dans les maisons de Varsovie remontées, pour partie seulement, avec les matériaux récupérés dans les ruines, voire totalement renouvelée: dans la réédification en béton et placage de la Frauenkirche de Dresde, scientifiquement fondée sur les archives historiques, les volumes ont été précisément reconstitués et le dessin exactement restitué, mais dans un matériau absolument hétérogène et totalement neuf ${ }^{40}$. Que dire alors du façadisme?

16 L'architecture japonaise ancienne, construite traditionnellement en bois, a porté ce débat à ses dernières extrémités ${ }^{41}$. On sait qu'au Japon, les édifices historiques ont constamment subi des restaurations de plus ou moins grande ampleur, voir des reconstructions totales après incendie. Ce souci de préservation et d'entretien a été à l'origine de formidables archives historiques et de la forme d'archéologie du bâti sans doute la plus ancienne du monde. Le cas du sanctuaire Shinto d'Isé est le plus remarquable. Les nombreux temples qui le composent sont renouvelés tous les vingt ans. Tous ces édifices restent inchangés dans leur plan, dans leur distribution à l'intérieur du téménos ${ }^{42}$, dans leur dessin architectural. Mais ils sont systématiquement renouvelés dans leur substance. La matière est toujours neuve, seule l'architecture est d'origine. Phénomène difficile à saisir pour un occidental, même si Chateaubriand voyait dans le sillon du laboureur un monument plus apte à atteindre l'éternité que les pyramides d'Égypte. Cette architecture "qui n'est chaque fois ni tout à fait la même, ni tout à fait une autre " (et qui par là rejoint la musique, se mouvant entre la stabilité de l'écriture et la volatilité de l'interprétation) a récemment légitimé la création par l'UNESCO de la catégorie de Patrimoine immatériel de l'humanité.

17 Vue du Japon, notre architecture peut donc apparaître comme un conservatoire de matériaux, justifié et imposé par la passion de l'authentique. L'authenticité est tout entière attachée à la préservation des matériaux d'origine, un peu à la manière des reliques du Moyen Âge : la puissance du saint, sa virtus, est efficiente parce que l'objet qui en est le support - ossements, vêtements, ustensiles... - lui a authentiquement appartenu (mais on admet aussi que cette virtus puisse se communiquer par contiguïté à des matériaux ayant été en contact avec les reliques: n'est-ce pas aussi le cas des restaurations ?). Cette attitude culturelle n'est pas dénuée de fondements : les matériaux 
d'origine portent la trace du travail des constructeurs et de leur pensée. Et c'est ces traces que l'archéologie du bâti analyse, pour restituer le chantier de construction dans tous ses aspects, projet, chronologie, transmission des savoir, changements de parti, inventions techniques ${ }^{43} \ldots$ Troublante relation au temps qui transparait dans toutes les communications de ces actes. Car le matériau travaillé appartient à la fois au passé, par l'action, et au présent, à notre présent, par sa permanence. Il est, pour reprendre la catégorie grammaticale du passé-composé, le résultat présent d'une action passée, à laquelle il nous donne directement, immédiatement accès. Ce n'est pas sans raison que le passé simple est le temps du récit : il exprime une action passée en elle-même et pour elle-même, totalement indépendante du présent. C'est le temps des archives et des sources écrites, closes sur elles-mêmes. C'est le temps de nos récits d'historiens de l'art.

L'architecture est donc à la fois un projet et un dessin matérialisé à l'échelle 1, tandis que l'architectonique en est la matérialisation en devenir. La seconde se présente comme une image en creux de la première. L'archéologie du bâti l'a démontré abondamment. Ce qui, en architecture, apparaît comme une file de fenêtres, se révèle, pendant la construction, être d'abord une suite de môles. Toutefois, il n'y a pas opposition, mais bien consubstantialité. Tout édifice est un dessin architectural, mais tout dessin architectural est une ligne matérialisée. L'évocation la plus limpide de cette consubstantialité du dessin et des matériaux, on la doit à un romancier. Dans Cités invisibles, Italo Calvino met en scène une discussion sur l'architecture entre Kubilaï Khan et Marco Polo : « Mais quelle est la pierre qui soutient le pont, demande Kubilaï Khan? Le pont n'est pas soutenu par cette pierre-ci ou celle-là, répond Marco, mais par la ligne de l'arc qu'elles forment. Kubilaï Khan garde le silence, pensif. Puis il ajoute : pourquoi me parles-tu des pierres ? C'est seulement l'arc qui m'importe. Polo répond: Sans pierres, il n'y a pas d'arc. »

\section{NOTES}

1. . En 1831, Jules Michelet notait dans son journal : «Ce qui me frappe à Caen [c'est-à-dire à la Société des antiquaires de Normandie], c'est que les mêmes hommes, Caumont, Laire, Vaultier, sont à la fois antiquaires et naturalistes. Mon compagnon de voyage confondait sans cesse histoire et histoire naturelle. C'est qu'en fait, Caen réunit d'une part les antiquités romaines et normandes, de l'autre les antiquités antédiluviennes, les fossiles, etc. » Cité par Alain Schnapp, La conquête du passé, Aux origines de l'archéologie, Paris, Éditions du Carré, 1993, p. 280.

2. En dernier lieu, voir Jean Nayrolles, L'invention de l'art roman à l'époque moderne (XVIII ${ }^{e}$-XIX siècles), Rennes, Presses Universitaires de Rennes, 2005, 402 p.

3. . Voir par exemple Les Veines du temps. Lecture de bois en Bourgogne, catalogue de l'exposition itinérante. CNRS, Musée Rolin. Autun, 1992, 454 p.

4. . Voir par exemple Enduits et mortiers, archéologie médiévale et moderne, Actes de la table ronde des 16-17 octobre 1987, dir. Christian Sapin, Dossier de documentation archéologique du CNRS n ${ }^{\circ}$ 15, 1991, 122 p. ; Daniel Prigent, « Méthodes d'investigations archéologiques utilisées à l'abbaye de Fontevraud », Fontevraud, histoire et archéologie, nº 4, 1995-1996, p. 17-43. 
5. . En dernier lieu, voir L'Homme et la matière, l'emploi du plomb et du fer dans l'architecture gothique, Actes du colloque de Noyon organisé par l'Agence Régionale du Patrimoine de Picardie (16-17 novembre 2006), Arnaud Timbert dir., à paraître, 2008.

6. . Pour une synthèse récente sur l'archéologie du verre architectural du Moyen Âge, voir Francesca Dell'Acqua, Illuminando collorat, la vetrata tra l'eta tardo imperiale e l'alto medioevo : le fonti, l'archeologia, Spoleto, 2003, 214 p.

7. . Voir en dernier lieu El ladrillo y su derivados en la época romana (Monografias de arquitectura romana 4), Manuel Bendala Galán, Chrristian Rico, Lourdes Roldán Gómez dir., Madrid 1999, 309 p.; Philippe Araguas, Brique et architecture dans l'Espagne médiévale, Madrid, Casa de Velasquez, 2003, 562 p.; Lombardini, « Hylè Historias, The Constructive Technologies in the church of Hagia Sophia at Constantinople and in the Building of Justinian's Reign ", Architectural-Structural Survey of Hagia Sophia, Kenichiro Hidaka et Tatsuki Sato, Chuo Koron Bijustsu Shuppan dir., $2004\left(1^{\mathrm{e}}\right.$ édition 2003), p. 286-301; James W. P. Cambell, L'Art et l'histoire de la brique, Paris, Citadelle et Mazenod, 2004, $320 \mathrm{p}$.

8. . Nicolas Reveyron, "L'apport de l'archéologie du bâti dans la monographie d'architecture », Analyse architecturale, la monographie d'architecture, séminaire sous la direction de Jean-Marie Pérouse de Montclos, Direction de l'architecture et du patrimoine, Paris, 26-27 avril 2000, In Situ, revue électronique de L'inventaire général, 2002.

9. Ces nouvelles orientations, qui concernent les aspects les plus « immatériels » du chantier, sont encore peu développées. L'étude du voûtement gothique de la cathédrale de Lyon, réalisé par des équipes de maçons locaux devant adapter leur savoir-faire vernaculaire à des techniques importées d'une autre culture technique, en offre un bon exemple.

10. . John Ruskin, The Seven Lamps of Architecture, Londres, Alice Meynell, 1849, 312 p.

11. . En dernier lieu, voir Patrick Boucheron, Le pouvoir de bâtir, urbanisme et politique édilitaire à Milan (XIV-XVe siècles), Rome, École française de Rome, 1998, 653 p.

12. . Dieter Kimpel, «Le développement de la taille en série et son rôle dans l'histoire économique », Bulletin Monumental, 1977, p. 195-222.

13. . Christiane Demeulenaere-Douyère, David-John Sturdy, L'Enquête du Régent, 1716-1718, Sciences, techniques et politiques dans la France préindustrielle, Turnhout, Brépols, 2008, 1018 p.

14. . Isabelle Parron-Kontis dir., La Cathédrale de Moûtiers en Tarentaise et le groupe épiscopal de Maurienne, Lyon, association lyonnaise pour la promotion de l'archéologie en Rhône-Alpes, 2002, $156 \mathrm{p}$.

15. . Salvatore Ruffolo, «Le strutture murarie degli edifici paleocristiani milanesi », Rivista del Istituto di Archeologia e Storia dell'Arte, XVII, 1972; M. Mirabella Roberti, «Edilizia e architettura ambrosiana a Milano ", XXV corso di cultura sull'arte ravennate e bizantina, Ravenne, 1978, p. 191 et suiv.

16. . Frédérique-Anne Constantini, L'Abbaye Saint-Robert de la Chaise-Dieu, un chantier de la Papauté d'Avignon (1344-1352), Paris, Honoré Champion, 2003.

17. . Pour une réflexion générale sur cette problématique récente, voir Jean-Marc Huygen, La Poubelle et l'architecte, Vers le réemploi de matériaux, Arles, Actes Sud, 2008, 184 p.

18. . Voir notamment Lucilla de Lachenal, Spolia, uso e reimpiego dell'antico dal III al XIV secolo, Milan, Longanesi, 1995, 442 p. ; Antike Spolien in der Architektur des Mittelalters und der Renaissance, Heausgeben von Joachim Poeschke, Munich, Hirmer Verlag, 1996, 368 p.

19. . «Marmoreas aut marmoreis aequipollentes haberemus columpnas ». Suger, CEuvres, tome I, texte établi, traduit et commenté par Françoise Gasparri, Paris, Les Belles Lettres, 1996, p. 13.

20. . Le terme choin désigne des blocs de remploi de très grands modules, taillés à l'époque antique dans un calcaire très dur (calcaire froid), blanc et susceptible d'être poli comme le marbre. La première mention conservée du terme, sous la forme chaon, est datée de 1192 (" marmorei lapides et illi qui vulgo chaon »). Marie-Claude Guigue, « Notice sur la construction de 
la cathédrale de Saint-Jean et ses chapelles ", Lucien Bégule, Monographie de la cathédrale de Lyon, Lyon, Mougin-Rusand, 1880, p. 4 ; Nicolas Reveyron, «Exploitation des pierres dans leurs potentialités techniques et esthétiques : l'exemple de Lyon au Moyen Âge ", Pierres du patrimoine européen, de l'extraction à la mise en ceuvre et à la valorisation, Actes du colloque international Château-Thierry (18-21 octobre 2005), à paraître (2008).

21. . Klaus Grewe, « Aquädukt-Marmor, Kalksinter der römischen Eifelwasserleitung als Baustoff des Mittelalters », Sonderdruk Bonner Jahrbüche, t. XCI, Stuttgart, 1992, p. 277-343.

22. . Cité par Philippe Guigon, Les Églises du haut Moyen Âge en Bretagne, Saint-Malo, centre régional d'archéologie d'Alet, t. II, 1997, p. 31 et suiv.

23. . La Gobertange, une pierre, des hommes, Freddy Damblon dir., Louvain, CERA Holding, 2000, 413 p.

24. . Voir en dernier lieu Laurent Dujardin, « Le commerce de la pierre de Caen (XI ${ }^{\mathrm{e}}-\mathrm{XVII}{ }^{\mathrm{e}}$ siècle) ", La Normandie dans l'économie européenne (XII $-\mathrm{XVII}{ }^{e}$ siècle), actes du colloque de Cerisy-la-Salle, octobre 2006, dir. Mathieu Arnoux, Anne-Marie Flambart-Héricher (à paraître).

25. Camille Enlart, Manuel d'archéologie française depuis les temps mérovingiens jusqu'à la Renaissance , I - Architecture religieuse, Paris, 1919 (édition augmentée), p. 89-90.

26. . Peter Cornelius Claussen, Magistri Doctissimi Romani, Die römischen Marmorkünstler des Mittelalters. Corpus cosmatorum, Wiesbaden-Stuttgart, Franz Steiner, 1987, 264 p.

27. . «Quod genus lapidum in Bononiensi provin[c]ia tantummodo inveniri dicitur [...] Naturaliter autem hii lapides in oriente apud Coloniensem provin[c]iam reperiuntur ». Victor Mortet, Recueil de textes relatifs à l'histoire de l'architecture et à la condition des architectes en France au Moyen Âge (XI ${ }^{e}$-XIII ${ }^{e}$ siècles), Paris, 1911, p. 172-173.

28. . « Ainz ne finerent, si vindrent a Vesene, / A Lavardi ou la pierre fu trete / Dont les toreles de Nymes furent fetes », La Mort de Roland, Domnique Boutet, tr., Paris, Le Livre de poche, 1996, vers 1055-1057, p. 262.

29. . Pierre et métal dans le bâtiment au Moyen Âge, Odette Chapelot, Paul Benoit dir., Paris, éd. de l'ÉHÉSS, 2001, 370 p. ; Du projet au chantier. Maîtrise d'ouvrage et maîtrise d'œuvre aux XIVe-XVI ${ }^{e}$ siècles , Paris, éditions de l'École des hautes études en sciences sociales, 2001, 604 p.

30. . Voir les actes des congrès nationaux des sociétés savantes, Jacqueline Lorentz dir., Carrières et constructions en France et dans les pays limitrophes, 1991, 1993, 1996, 2004.

31. . Voir par exemple Alain Salamagne, «L'approvisionnement en pierre des chantiers médiévaux : l'exemple de Douai (Nord) aux XIV et XV siècles", Archéologie médiévale, 1997, p. 45-76.

32. . Sur les traces de taille, les modes d'analyse et leur application à l'étude du bâti, voir Nicolas Reveyron, avec la collaboration de Ghislaine Macabéo et les contributions de Christian Le Barrier et Hervé Chopin, Chantiers lyonnais du Moyen Âge, Saint-Jean, Saint-Nizier, Saint-Paul, archéologie et histoire de l'art, Lyon, Documents d'archéologie Rhône-Alpes, Lyon, association lyonnaise pour la promotion de l'archéologie en Rhône-Alpes, 2005, 384 p.

33. En 1902, Camille Enlart (Manuel d'archéologie française depuis les temps mérovingiens jusqu'à la Renaissance, I - Architecture religieuse, Paris, Auguste Picard, 1902, 477 p.) proposait une première chronologie du phénomène, des sarcophages mérovingiens aux églises germaniques du XIII ${ }^{\mathrm{e}}$ siècle.

34. . N. Reveyron, "Marques lapidaires : the State of the Question ", Gesta, t. XLII, n 2, 2003, p. 271-311.

35. . Sur tous ces points, voir Nicolas Reveyron, "Support-surface», Actes du $X^{e}$ colloque international de glyptographie, centre international de glyptographie - Mont-Sainte-Odile, 4-9 juillet 1996, Braine-le-château, 1997, p. 427-444.

36. . Éric Palazzo, Liturgie et société au Moyen Âge, Paris, Aubier, 2000, 276 p.

37. . Au XIII ${ }^{\mathrm{e}}$ siècle déjà, l'évêque de Lausanne était accusé d'avoir détourné du chantier de la cathédrale des pierres pour son usage personnel. Jean-Charles Biaudet, Henri Meylan, Werner 
Stöckli, Philippe Janton, Marcel Grandjean, Claude Lapaire, Ellen Judith Beer, La Cathédrale de Lausanne, Berne, Société d'histoire de l'art en Suisse, 1975, 264 p.

38. . Paul Veyne, Comment on écrit l'histoire, Paris, Le Seuil, Points-histoire, 1996, p. 211.

39. . Nicolas Reveyron, "Réflexions sur la place des savoir-faire dans la perception du beau architectural », Siècles, cahiers du centre d'histoire « Espaces et culture », 22-2005, p. 23-38.

40. . Depuis les réflexions pionnières d'Alois Riegl (Der moderne Denkmalkultus, sein Wesen und seine Entstehung, Vienne, 1903), les problématiques concernant les matériaux, l'archéologie du bâti, la restauration, la conservation, la mémoire culturelle et le patrimoine monumental se sont trouvées inextricablement liées. Citons notamment Peter Kurmann, Restauriegung, Retrospektive, Rezeption, Retardierung und Rekonstruktion: "Gedanken zur Denkmalpflege anhand historischer und zeitgenössischer Beispiele", Geschichte der Restauriegung in Europa, t. I, Worms, 1991, p. 14-28 ; Faut-il restaurer les ruines?, Actes des Entretiens du Patrimoine, Paris, direction du patrimoine, 1991, 225 p.; H. Wirth, Werte und Bewertung baulich-räumerlich Strukturen. Axiologie der baulichräumerlichen Umwelt, Alfter, 1994 ; "Conservation, restauration : doctrine », Monumental, t. XXII, 1998 ; L'Abus monumental ?, actes des $4^{e}$ Entretiens du patrimoine, Paris, Fayard, 1999, 400 p. ; Camillo Boito, Conserver ou restaurer. Les dilemmes du patrimoine, Besançon, Éditions de l'imprimeur, 2000, 112 p. ; La Forteresse à l'épreuve du Temps, Destruction, dissolution, dénaturation, $X I^{e}-X X^{e}$ siècle, Gilles Blieck, Philippe Contamine, Christian Corvisier, Nicolas Faucherre et Jean Mesqui dir., Paris, CTHS, 2007, $334 \mathrm{p}$.

41. . Voir notamment Masatsugu Nishida, "Time in Japanese Architecture, A Reflexion through the Practice of Reconstruction », Design Discours, vol. 1, nº 1, 2005, p. 9-23 ; Jean-Sébastien Cluzel, Masatsugu Nishida, Philipe Bonnin, " "Authenticité" et reconstruction de la mémoire dans l'architecture monumentale japonaise ", Espace et société, 2007-4, 131, p. 153-170 ; Jean-Sébastien Cluzel, Masatsugu Nishida, «Le temps des monuments face au temps de l'histoire », Bulletin de l'École française d'Extrême-Orient, à paraître. J'ai plaisir à remercier ici le professeur Nishida de m'avoir fourni une très riche bibliographie et de m'avoir fait découvrir trois semaines durant les richesses monumentales du Japon médiéval.

42. . Chaque temple occupe un vaste terrain, entouré de palissades et flanqué d'un terrain de mêmes dimensions, qui est préparé pour la reconstruction du temple. Quand le temple est (ré)édifié sur ce second terrain, l'ancien terrain est déblayé et remis aux normes. Vingt ans plus tard, le temple est à nouveau construit sur ce premier terrain. Et ainsi de suite. Ce mouvement de bascule systématique équivaut à une véritable stabilité spatiale.

43. . Nicolas Reveyron, "L'apport de l'archéologie du bâti dans la monographie d'architecture », Analyse architecturale, la monographie d'architecture, séminaire sous la direction de Jean-Marie Pérouse de Montclos, In Situ, revue électronique de L'inventaire général, 2002 ; id., " Histoire de l'architecture et archéologie du bâti : l'interdisciplinarité au cœur de l'édifice ", Repenser les limites : l'architecture à travers l'espace, le temps et les disciplines, colloque international de Paris (31 août-4 septembre 2005, INHA Paris - Society of Architectural Historians de Chicago), à paraître.

\section{RÉSUMÉS}

Depuis la fin du siècle dernier, les matériaux de construction se sont retrouvés au centre de la recherche en histoire de l'architecture. Textes et archives témoignent de l'importance qu'ils ont pris dès le Moyen Âge (et bien avant) dans l'imaginaire occidental. Les jugements esthétiques, les 
correspondances des acteurs du chantier, les ordres du commanditaire, les dessins des plans et des élévations, les factures et les prix-faits, les polémiques dont se font écho la poésie, les nouvelles, les romans ou les articles de journaux, tous ces documents jettent, chacun à sa manière, des lumières très diverses sur les pierres de l'architecture, du projet et de l'extraction à la mise en œuvre et aux finitions. L'archéologie puise dans les pierres du bâti une masse considérable de renseignement sur le chantier et la chronologie des travaux, grâce à l'étude notamment des traces du travail (empreintes laissées par les outils, vestiges des savoir-faire, erreurs et repentirs...), des marques lapidaires, des dimensions des blocs, de la géologie du monument etc. Les matériaux se trouvent aussi à la conjonction de la technique, de l'esthétique, que les siècles ont fait évoluer jusqu'à la rendre méconnaissable, et de notre culture. En d'autres termes, à la conjonction du passé et de notre présent, du dessin et de la matière. Or, justement, les relations entre architecture et matériaux sont loin d'avoir l'évidence qu'elles laissent paraître.

Since the end of the last century, researches on building materials are at the very centre of architectural history studies. Texts and archives show how important they were in the imagery of the Occident in the Middle Ages (and far before). These documents - aesthetic texts and judgments, letters written by the building process' participants, orders given by patrons, plans and elevations' drawings, invoices and contracts, polemics in poetry texts, in shorts stories and novels or in newspapers - throw different kind of lights on the stones of architecture and on all the process, from the project and the quarrying to the building fabric and the finishing touches. We can collect a large amount of information on the building work and its process, thanks to archaeological studies, i.e. works marks (tool tracks, faults and regrets...), lapidary marks, stone blocks' size, etc. Building materials are therefore at the crossroads of the past and our present time, of drawing and material. Precisely, ties between architecture and building materials are not so obvious as they could seem at first glance.

Seit dem Ende des letzten Jahrhunderts erweisen sich die Baumaterialien als ein zentrales Forschungsthema der Architekturgeschichte.Texte und Archive zeugen also von der hochwichtigen Bedeutung der Baumaterialien ab dem Mittelalter (oder schon viel früher) in der allgemeinen Vorstellung der westlichen Welt. Unter diesen Dokumenten findet man ästhetische Beurteilungen, Briefwechsel zwischen den Akteuren der Baustelle, Aufforderungen der Auftraggeber, Grund- und Aufrisszeichnungen, Rechnungen und Kostenvoranschläge sowie das literarische Echo von erregten Polemiken in Novellen, Romanen oder Zeitungsartikeln. Dadurch werden die Grundsteine und Etappen der Architektur ins Licht gerückt, von dem Projekt bis zur Erstellung. Andererseits schöpft die Archäologie aus der Untersuchung der Bausteine eine Menge von Informationen über die Chronologie der Bauarbeiten, ebenso beim Sammeln von Arbeitsspuren (Steinhauerzeichen, hinterlassene Zeichen der damaligen Handwerkskunst ebenso wie Fehler und Abänderungen ).Die Beobachtung der Steinzeichen, der Dimension und der geologischen Natur der Steinblöcke erweist sich auch besonders lehrreich. Zwischen Technik und Ästhetik zeugen die Baumaterialien offensichtlich auch von den kulturellen Veränderungen im Laufe der Jahrhunderte.

\section{AUTEUR}

\section{NICOLAS REVEYRON}

Nicolas Reveyron, agrégé de lettres classiques, docteur en histoire de l'art de la Sorbonne et archéologue spécialiste du bâti, Nicolas Reveyron est professeur d'histoire de l'art et archéologie du Moyen Age à l'Université Lumière-Lyon 2 et membre de l'Institut Universitaire de France. Il 
mène des recherches sur l'architecture religieuse médiévale, le chantier de construction et l'image des édifices médiévaux dans les textes et les arts figuratifs. Principales publications : Nicolas Reveyron, Véronique Rouchon, L'Art roman, Paris, Flammarion, collection "Abécédaire", 2000 ; Anne Prache, Philippe Plagneux, Nicolas Reveyron, Daniel V. Johnson dir., Initiation à l'art roman, Paris, Desclée de Brouwer-Zodiaque, 2002 ; Jean-Noël Barnoud, Nicolas Reveyron, Gilles Rollier, La Basilique de Paray-le-Monial, Paris, Desclée de Brouwer-Zodiaque, 2004 ; Nicolas Reveyron, Chantiers lyonnais du Moyen Âge, Saint-Jean, Saint-Nizier, Saint-Paul, archéologie et histoire de l'art, Lyon, Documents d'Archéologie Rhône-Alpes, Lyon n 28, 2005 ; Nicolas Reveyron, L'Art roman, Paris, Editions du Cavalier Bleu, 2008. 